PROCEEDINGS of the Tenth International Driving Symposium on Human Factors in Driver Assessment, Training and Vehicle Design

\title{
UNDERSTANDING LANE-KEEPING ASSIST: DOES CONTROL INTERVENTION ENHANCE PERCEIVED CAPABILITY?
}

\author{
John M. Sullivan, Michael J. Flannagan \\ Human Factors Group \\ The University of Michigan Transportation Research Institute \\ 2901 Baxter Road \\ Ann Arbor, MI 48109 \\ E-mail: jsully@umich.edu
}

\begin{abstract}
Summary: Drivers of vehicles equipped with ADAS often show a flawed understanding of the limitations of these systems. In this study, two types of lane keeping assist (LKA) were investigated: a lane centering system that continuously repositioned the vehicle in the center of the lane, and a lane departure prevention system that intervened when the vehicle wandered near the lane edge. Driver knowledge of each LKA (and accompanied ACC) were tested over a series of five drives. Results suggest that greater capability may be attributed to the lane centering system, perhaps because its control intervention is more frequent and obvious than the lane departure prevention LKA.
\end{abstract}

\section{INTRODUCTION}

Advanced driver assistance systems (ADAS) present a growing opportunity to improve driver safety by alerting drivers to dangerous conditions and relieving drivers of tedious control tasks through automation. However, the actual effectiveness of these systems often depends on how drivers adapt their driving behavior to these systems, which in turn depends on how well drivers understand these systems. Current ADAS technologies include operating characteristics that often violate driver expectations or, at best, do not conform to a driver's mental model of the system. Several studies have documented instances in which drivers fail to recognize that an ADAS is functioning improperly. For example, in simulator studies, drivers equipped with adaptive cruise control (ACC) were found to be generally slower than drivers with manual control to react to critical traffic situations. These includes abrupt lead vehicle braking, vehicle cut-ins, the sudden reveal of stationary vehicles in the road, and system failures (e.g., de Winter, Happee, Martens, \& Stanton, 2014; Hoedemaeker \& Brookhuis, 1998; Young \& Stanton, 2007)

While sometimes explained in terms of behavioral adaptation (Sullivan, Flannagan, Pradhan, \& Bao, 2016), drivers often appear to overgeneralize ADAS capabilities and they do not appear to remember operational exceptions (even if told) unless they are given more direct experience of the exception (Beggiato, Pereira, Petzoldt, \& Krems, 2015). It is possible that when a driver is first exposed to an ADAS, he or she may try to match it to prior experience with a similar form of intelligent support - maybe even another human acting as an operator. This suggests that drivers may require time to fine tune their understanding of an ADAS, especially when the conditions where the ADAS's limitations can be observed are also uncommon.

This study focuses on a driver's understanding of two ADAS technologies: speed assistance or ACC, and steering assistance, or lane keeping assistance (LKA). Prior research on ACC suggests that drivers are unlikely to remember "exception" conditions or when the ADAS reaches some kind of performance limit, unless there is more direct experience with this condition (Beggiato \& Krems, 2013; Beggiato et al., 2015; Bianchi Piccinini, Rodrigues, Leitão, \& Simões, 2015). 
When a driver's principal source of information about the operation of an ADAS is the owner's manual, and boundary conditions are unlikely to be encountered during normal driving experience, it seems clear that gaps in a driver's understanding of the system will commonly occur (Dickie \& Boyle, 2009; Jenness, Lerner, Mazor, Osberg, \& Tefft, 2008). More troubling is that gaps may also arise, as memories fade and are not reinforced by experience. Such knowledge gaps may result in the driver developing unrealistic expectations about the technology's capability and leave drivers unprepared to take control when the technology falters (Merat, Jamson, Lai, Daly, \& Carsten, 2014).

This study identifies another characteristic that may influence a driver's perception of an ADAS's capability - the degree to which an ADAS's activity is obvious to the driver. Two forms of lane keeping assistance (LKA) systems were investigated in this study: lane centering control, in which the lane keeping function centers the vehicle in the lane; and lane departure prevention, in which the lane keeping function intervenes when the vehicle approaches a lane boundary without signaling a lane change. Lane centering interventions are continuous as long as lane boundaries are detectable and the function is active; lane keeping assist interventions occur sporadically - when the vehicle nears a lane boundary. While the activation state of both systems is usually indicated on the instrument cluster, the lane centering system consistently makes its presence felt by the gentle steering torque regularly applied to correct any drift in the lane.

Drivers' understanding of two samples of an ACC and LKA technology were probed over a series of 1.5-hour test drives in one of two late model vehicles. One vehicle was equipped with a lane-centering LKA, the other with a lane departure LKA. Each driver took five test drives over a five-week period and, after each, their understanding of each system was probed.

\section{METHOD}

\section{Participants}

Participants in this study were recruited from the local licensed driver population. Two age groups were selected: an older-driver group between 60 to 70 years of age (mean, 64.2 years), and a young/middle-aged group between 20-50 years or age (mean, 32.0 years). Each age group was divided evenly by gender. Thirty-two drivers were recruited for the study. One driver dropped out after three driving sessions.

\section{Materials}

Surveys. Participants were given three surveys to complete. An initial survey was used to obtain general demographic information about each driver, their driving habits, and their general level of familiarity with in-vehicle support technologies. This survey was administered at the beginning of the study. Two other surveys were administered after each drive session that probed drivers' knowledge of the vehicle's ACC and LKA systems - called the ACC Survey and the LKA Survey. These two surveys are the focus of this study.

To assess each driver's knowledge about the ADAS functions, specific questions were asked about circumstances in which the ADAS might not be fully functional, but where drivers often fail to recognize such ADAS limitations. For ACC, drivers often show limited awareness that motorcycles, pedestrians, or bicycles may not be detected; that ACC may not react to slow-moving or stationary vehicles; that alignment problems on curvy roads may result in false readings; or that the ACC radar may be limited in active weather. The diagnostic questions developed for this study were based on questions used by Beggiato (2015) in his thesis that examined driver 
understanding of ACC. The questions that targeted a driver's understanding of ACC operation were as follows:

Rate your level of agreement with the following statements (using 5 levels of agreementstrongly disagree, somewhat disagree, neither, somewhat agree, strongly agree):

- The ACC detects all sizes of vehicle ahead of me

- The ACC can help boost my braking in an emergency

Rate how well ACC is suited to these traffic conditions (using 5 levels of appropriateness-extremely inappropriate, inappropriate, neither, appropriate, extremely appropriate):

- When you encounter a stopped vehicle in the roadway

- On curvy roadways

- Along roadways with bicycles and pedestrians present

- In snowy or rainy conditions

Other questions were developed to probe understanding of the steering assist system (LKA). As with the ACC questions, drivers were asked to rate how well the LKA would deal with different traffic situations using the same 5-point rating of appropriateness.

- Construction zones. Poorly marked lanes adversely affect LKA operation.

- Very curvy roadways. Steering authority of LKA may be exceeded on curved roads.

- Snow-covered and rainy weather. Snow and rain can obscure lane markings.

- Responding to objects in the roadway. LKA does not respond to debris in the roadway.

- Responding to potholes. LKA is not sensitive to potholes.

- Roadways with bicycles and pedestrians. LKA tracks lane boundaries, not other objects.

The surveys also contained other questions related to operational state confusion, unexpected intervention, and failures to intervene. These will not be discussed here.

Vehicles. Two late-model vehicles equipped with steering assist and ACC were selected for use in this study. The models differed in size and implementation of their ADAS functions as shown in Table 1. Model A was a compact sedan priced at the lower-end of the vehicle market. Model $\mathrm{B}$ was a mid-sized sedan priced about $\$ 10,000$ more than Model A. The two models were chosen for their full speed range of support for cruise control as well as the differences between their steering assist systems. Model A's steering assist provided continuous lane centering control (LCC) and asserted control continuously during the drive as long as lane markings were detectable on the roadway. Model B's steering assist intervened only after the vehicle was detected at (or near) the lane boundary. An informal impression was that Model B's steering intervention was relatively subtle, compared to Model A.

\section{Procedure}

Participants were recruited to drive one of the advanced technology vehicle models for approximately 1.5 hours along a prescribed route, once a week, for five drive sessions. In the first drive session, all drivers were given a consent form that detailed the study. Each driver was assigned to drive either the Model A or Model B vehicle and provided with excerpts from the owner's manuals of each respective vehicle describing the operation of each vehicle's ACC and LKA system. Assignment of drivers to vehicles were balanced by age and gender. Drivers were given as much time as they required to review the orientation materials and asked to use the ACC and LKA as often as they felt appropriate. Thus, the experimental design involved three between-subjects factors - age group (middle, old), gender (male/female), vehicle model (A/B) — and one within 
subject factor, drive session number (1-5). Dependent measures were participant's judgments about the function of each system measured by the repeated surveys.

Drivers were accompanied by experimenters seated in the rear of the vehicle. Experimenters provided route guidance to participants, but did not provide any further information about the function of the ACC or LKA systems. Participants were permitted to consult the owner's manual after pulling off the road and stopping the vehicle. The overall drive was about a 40 -mile round trip along a 2-lane divided highway with posted speed limits of $70 \mathrm{mph}$.

Table 1. Comparative attributes of the two vehicle models

\begin{tabular}{|c|c|c|}
\hline Attribute & Model A & Model B \\
\hline \multirow[t]{2}{*}{ Adaptive Cruise Control } & 4 following distances & 3 following distances \\
\hline & $\begin{array}{l}\text { Low speed down to stop-full speed } \\
\text { range ACC. }\end{array}$ & $\begin{array}{l}\text { Low speed down to stop-full speed } \\
\text { range ACC. }\end{array}$ \\
\hline Steering Assist & $\begin{array}{l}\text { Keeps vehicle centered in lane-Lane } \\
\text { Centering Control (LCC) }\end{array}$ & $\begin{array}{l}\text { Returns vehicle to lane on detection of } \\
\text { lane boundary-Lane Keeping Assist } \\
\text { (LKA) }\end{array}$ \\
\hline Size Class & Compact & Mid-size \\
\hline Max Weight & 2,923 lbs. & 3,388 lbs. \\
\hline Price (approximately) & $\$ 21,000$ & $\$ 30,000$ \\
\hline
\end{tabular}

\section{RESULTS}

Survey ratings were converted to numeric scales reflecting appropriateness and analyzed using linear mixed effects models in which participant was modeled as a random effect and gender, age group, and vehicle model were modeled as fixed effects. The principal analyses focused on answers to those questions that participants were most likely to answer incorrectly if the capabilities of the ADAS were either overestimated or overgeneralized. Participant responses to the ACC questions are reviewed first, followed by analysis of responses to the LKA questions. The analyses used the lme4 package of the R statistical programing package (Bates, Machler, Bolker, \& Walker, 2015) to perform a linear mixed effects analysis of the relationship between gender, age group, vehicle model, session number on each dependent mental model measure. Results of these analyses are summarized by technology and specific knowledge item.

\section{Beliefs about ACC}

ACC sees all sizes of vehicles. On average, most participants were in high agreement that both ACC systems could see all sizes of vehicles. On a scale of 1 to 5 where 5 is maximum agreement, the average agreement level was $4.5(\mathrm{SE}=0.25)$. No effect of session, gender, age, or mode were observed.

$A C C$ boosts braking. An effect of gender was observed $\left(F_{(1,26)}=4.5, p=.04\right)$. Male drivers agreed that ACC could help boost braking in an emergency $(\mathrm{M}=3.9, \mathrm{SE}=0.22)$ more than female drivers $(\mathrm{M}=3.19, \mathrm{SE}=0.23)$, with an average difference in agreement of about 0.7 units $(t=2.21, p=.04)$. No other effects of age, model, or session were observed.

Use of ACC is appropriate around stopped vehicles. A main effect of vehicle model was observed $\left(F_{(1,26)}=4.87, p=.04\right)$ such that Model A was rated more appropriate than Model B by about 0.83 units $(t=2.05, p<.05)$. This is shown in Figure 1a. No other effects were observed.

Use of ACC on curvy roadways. A main effect of vehicle model was observed such that the use of the ACC of Model A was rated as more appropriate on curvy roads $\left(F_{(1,26)}=10.7, p<.01\right.$; 
$\mathrm{M}=3.5, \mathrm{SE}=0.21)$ than Model $\mathrm{B}(\mathrm{M}=2.5, \mathrm{SE}=0.20)$ differing by about 1 rating unit $(t=3.27$, $p<.01$; shown in Figure 1b).

Use of ACC on roadways with pedestrian and bicycles. No main effects were observed in rated appropriateness. Mean ratings were below the neutral point of the scale $(2.78, \mathrm{SE}=0.34)$.

Use of ACC in bad weather. No main effects were observed in rated appropriateness of ACC use in bad weather. As before, the average ratings were at the neutral point (3.0, $\mathrm{SE}=0.36)$.
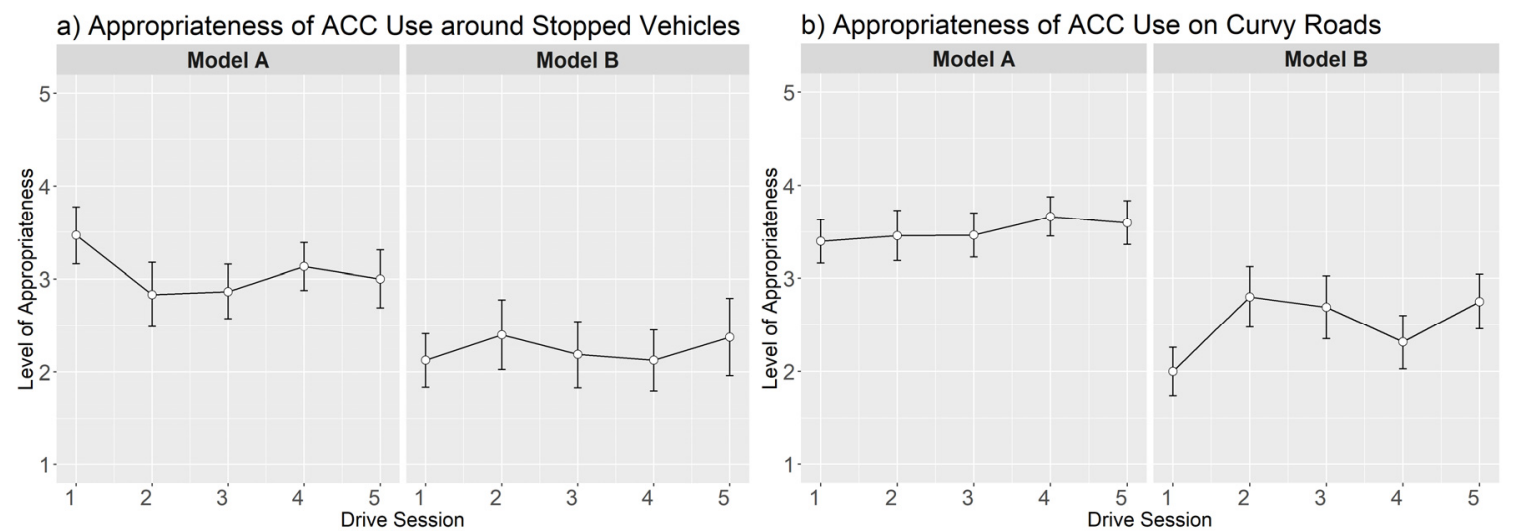

Figure 1. Rated appropriateness of use of ACC around stopped vehicles over each drive session (a); and on curvy roadways (b)

\section{Beliefs about LKA}

Use of LKA in construction zones. An effect of session was observed in drivers' rating of appropriateness of use of LKA around construction areas $\left(F_{(1,119)}=11.07, p<.01\right)$. In general, appropriateness declined by about 0.15 ratings points over each session Figure a).

Use of LKA on curvy roadways. An effect of vehicle model was observed in drivers' rating of appropriateness of LKA use on curvy roadways $\left(F_{(1,27)}=8.09 ; p<.01\right)$; Model A was rated 1.2 units more appropriate on curved roads than Model B (Figure b).

Use of LKA in snowy or rainy weather. For this measure, a gender effect was observed $\left(F_{(1,27)}=\right.$ 4.45; $p<.05)$ such that male drivers rated the appropriateness of LKA use in inclement weather about 0.6 units higher $(\mathrm{M}=2.8, \mathrm{SE}=0.21)$ than female drivers $(\mathrm{M}=2.2, \mathrm{SE}=0.22)$.

Use of LKA around objects, potholes, cyclists and pedestrians. For objects, effects of both vehicle model and session were observed (model $\left.F_{(1,27)}=6.15, p<.05 ; F_{(1,116)}=9.8, p<.01\right)$; for potholes, similar effects of model and session were also observed (model $F_{(1,27)}=6.36, p<.05$; session $\left.F_{(1,119)}=5.8, p<.05\right)$; and an effect of session was observed for pedestrians and bicycles $\left(F_{(1,119)}=10.59, p<.01\right)$ These effects are shown in Figure a, 3b, and 3c.

\section{DISCUSSION}

The results appear to show no influence of session on a driver's agreement or judgment appropriateness for ACC. Indeed, drivers appear to strongly agree that ACC is capable of detecting all vehicles throughout all sessions, despite the clear statement in the respective owner's manuals that ACC is limited in its ability to detect small vehicles. We suspect that the experience of the ACC continuously managing forward headway is overgeneralized as having the capability to detect all forward objects. The ACC results also show vehicle model effects, even though the two models' ACC systems are similar. Perhaps drivers' judgment of ACC capability is influence by 
their perception of LKA capability. The results seem to show that drivers systematically perceived Model A's ADAS technologies to be more capable of seeing objects, handling curves, operating in construction zones, and slightly more appropriate to use on roadways with objects, potholes, and pedestrians than Model B's ADAS. Drivers' confidence in Model A's ADAS technology may be influenced by these drivers' greater familiarity with the frequent lane-centering interventions of the LKA system. Such intervention may not only raise a driver's awareness of the system, but also lead to a broad elevation of confidence in the system and perhaps an overestimation of capability.
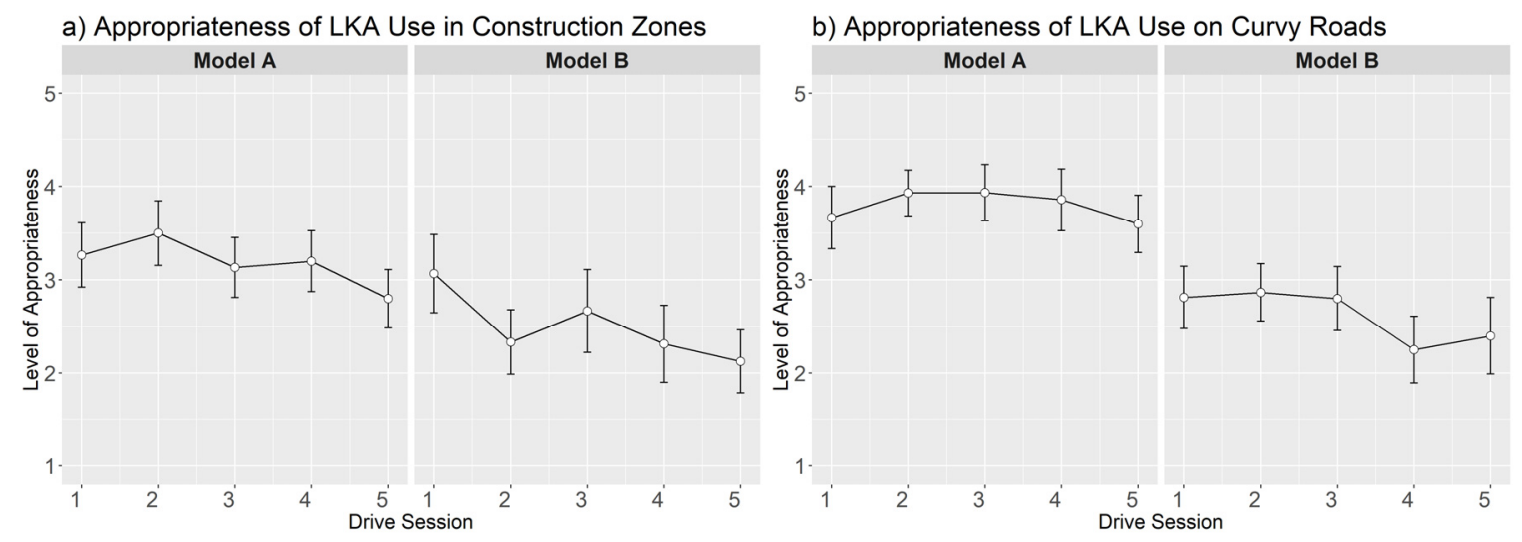

Figure 2. Judged appropriateness of LKA in construction areas (a) and on curvy roads (b)

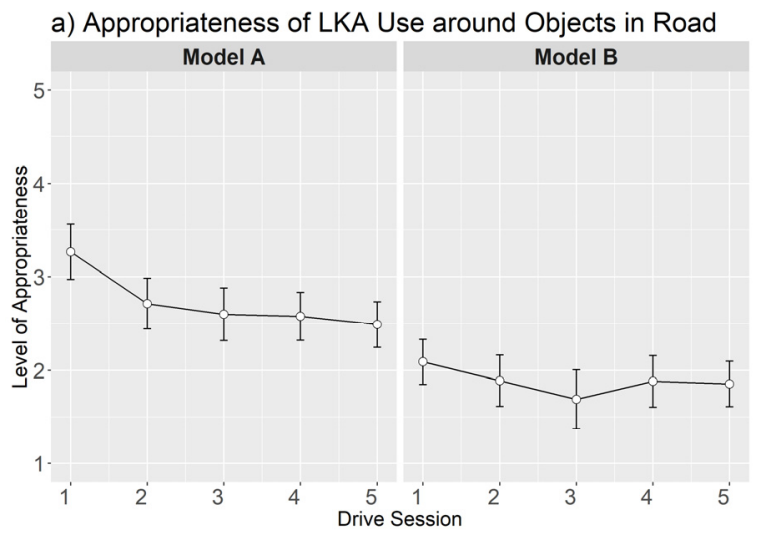

Figure 3. Declines in judged appropriateness of LKA around objects (a), potholes (b), and pedestrians and cyclists (c)

\section{ACKNOWLEDGEMENT}

This work was funded in part by the Toyota Class Action Settlement Safety Research and Education Program. The conclusions being expressed are the authors' only, and have not been sponsored, approved, or endorsed by Toyota or plaintiffs' class counsel.
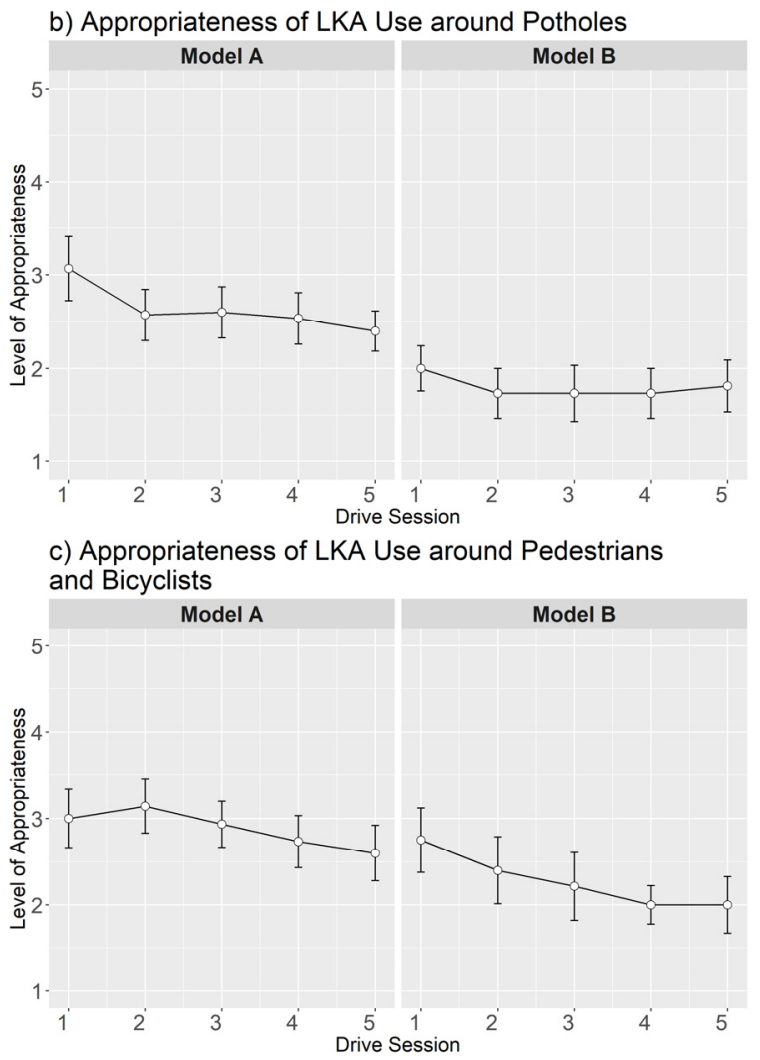


\section{REFERENCES}

Bates, D., Machler, M., Bolker, B. M., \& Walker, S. C. (2015). Fitting Linear Mixed-Effects Models Using lme4. Journal of Statistical Software, 67(1), 1-48.

Beggiato, M. (2015). Changes in motivational and higher level cognitive processes when interacting with in-vehicle automation. (Dr. rer. nat.), Technische Universität Chemnitz, Chemnitz.

Beggiato, M., \& Krems, J. F. (2013). The evolution of mental model, trust and acceptance of adaptive cruise control in relation to initial information. Transportation Research Part F, 18, 47-57.

Beggiato, M., Pereira, M., Petzoldt, T., \& Krems, J. (2015). Learning and development of trust, acceptance and the mental model of ACC. A longitudinal on-road study. Transportation Research Part F, 35, 75-84.

Bianchi Piccinini, G. F., Rodrigues, C. M., Leitão, M., \& Simões, A. (2015). Reaction to a critical situation during driving with Adaptive Cruise Control for users and non-users of the system. Safety Science, 72, 116-126.

de Winter, J. C. F., Happee, R., Martens, M. H., \& Stanton, N. A. (2014). Effects of adaptive cruise control and highly automated driving on workload and situation awareness: A review of the empirical evidence. Transportation Research Part F, 27, Part B, 196-217.

Dickie, D. A., \& Boyle, L. N. (2009). Drivers' Understanding of Adaptive Cruise Control Limitations. Proceedings of the Human Factors and Ergonomics Society Annual Meeting, 53(23), 1806-1810.

Hoedemaeker, M., \& Brookhuis, K. A. (1998). Behavioural adaptation to driving with an adaptive cruise control (ACC). Transportation Research Part F, 1, 95-106.

Jenness, J. W., Lerner, N. D., Mazor, S., Osberg, J. S., \& Tefft, B. C. (2008). Use of Advanced In-Vehicle Technology by Young and Older Early Adopters. Survey Results on Adaptive Cruise Control Systems (DOT HS 810-917). National Highway Traffic Safety Administration.

Merat, N., Jamson, A. H., Lai, F. C. H., Daly, M., \& Carsten, O. M. J. (2014). Transition to manual: Driver behaviour when resuming control from a highly automated vehicle. Transportation Research Part F, 27, Part B(0), 274-282.

Sullivan, J. M., Flannagan, M. J., Pradhan, A. K., \& Bao, S. (2016). Literature Review of Behavioral Adaptation to Advanced Driver Assistance Systems. Washington, D.C.: AAA Foundation for Traffic Safety.

Young, M., \& Stanton, N. A. (2007). What's skill got to do with it? Vehicle automation and driver mental workload. Ergonomics, 50(8), 1324-1339. 